\title{
sciendo
}

Int. J. of Applied Mechanics and Engineering, 2018, vol.23, No.3, pp.689-705

DOI: $10.2478 /$ ijame-2018-0038

\section{COMBINED EFFECTS OF SORET AND DUFOUR ON MHD FLOW OF A POWER-LAW FLUID OVER FLAT PLATE IN SLIP FLOW RIGIME}

\author{
K. SARITHA \\ Department of Sciences, Teegala Krishna Reddy Engineering College \\ Meerpet, Hyderabad-500079, Telangana, INDIA \\ M.N. RAJASEKHAR \\ Department of Mathematics, JNTUH College of Engineering \\ Nachupally, Karimnagar-505501, Telangana, INDIA \\ B.S. REDDY ${ }^{*}$ \\ Department of Sciences and Humanities \\ Sreenidhi Institute of Science and Technology \\ Yamnampet, Ghatkesar, Hyderabad-500301, Telangana, INDIA \\ E-mail: bsreddy_shashi@yahoo.com
}

\begin{abstract}
A numerical model is developed to study the Soret and Dufour effects on MHD boundary layer flow of a power-law fluid over a flat plate with velocity, thermal and solutal slip boundary conditions. The governing equations for momentum, energy and mass are transformed to a set of non-linear coupled ordinary differential equations by using similarity transformations. These non-linear ordinary differential equations are first linearized using a quasi-linearization technique and then solved numerically based on the implicit finite difference scheme over the entire range of physical parameters with appropriate boundary conditions. The influence of various governing parameters along with velocity, thermal and mass slip parameters on velocity, temperature and concentration fields are examined graphically. Also, the effects of slip parameters, the Soret and Dufour number on the skin friction, Nusselt number and Sherwood number are studied. Results show that the increase in the Soret number leads to a decrease in the temperature distribution and to an increase in concentration fields.
\end{abstract}

Key words: power-law fluid, Soret and Dufour number, slip boundary conditions, finite difference method, Prandtl number.

\section{Introduction}

It is well known that most fluids which are encountered in chemical and allied processing applications do not satisfy the classical Newton's law of viscosity and are accordingly known as nonNewtonian fluids. The fluids which do not obey Newton's law of viscosity, i.e., there exists a non-linear relationship between shear stress and velocity gradient are called non-Newtonian fluids. The study of nonNewtonian fluid has been of much interest to scientists because some industrial materials are nonNewtonian. In some industries (such as in food, polymer, petrochemical, rubber, paint and biological industries) fluids with non-Newtonian behaviors are encountered. The most common type of non-Newtonian fluids are power law fluids for which the shear stress $\tau$ is given by

\footnotetext{
${ }^{*}$ To whom correspondence should be addressed
} 


$$
\tau=\mu\left(\frac{\partial u}{d y}\right)=\mu_{0}\left(\frac{\partial u}{d y}\right)^{n} \quad \text { where } \quad \mu=\mu_{0}\left(\frac{\partial u}{d y}\right)^{n-1}
$$

where $\mu_{0}$ is the dynamic coefficient of viscosity is, $\frac{\partial u}{\partial y}$ is the shear rate and $n$ is the power-law index. It needs to be mentioned that, for a non-Newtonian power law model, the case of $n<1$ is associated with pseudo-plastic fluids (shear thinning fluids), $n=1$ corresponds to Newtonian fluids and $n>1$ applies to the case of dilatants fluids (shear thickening fluids).

The layer of the fluid that flows directly adjacent to its bounding surface is called the boundary layer. The boundary layer is an extremely important concept in fluid mechanics and has been studied extensively for decades. Fluid motion in the boundary layer is influenced by a number of factors namely fluid viscosity, external forces, bounding surface characteristics and so on. Sakiadas [1] was the first to consider the boundary layer flow at a continuous solid surface with constant speed. Crane [2] extended the work of Sakiadas [1] to the flow caused by an elastic sheet moving in its own plane with a velocity varying linearly with the distance from a fixed point. This work was later extended by many authors [3, 4, 5] by considering the flow, heat and mass transfer under different physical situations. Historically, the boundary layer flow past a flat plate was first considered by Blasius [6] to illustrate the application of Prandtl's boundary layer theory. Ali [7] discussed the problem of coupled heat and mass transfer by natural convection from a vertical impermeable semi-finite flat plate embedded in a non-uniform non-metallic porous medium in the presence of thermal dispersion effects. Pop and Takhar [8] studied thermal convection near a partly insulated vertical flat plate embedded in a saturated porous medium. These investigators, however, restricted their analyses to the flow of Newtonian fluids.

The theory of non-Newtonian fluids offers mathematicians, engineers and numerical specialists varied challenges in developing analytical and numerical solutions for highly non-linear equations. However, due to the practical significance of these non-Newtonian fluids, many authors have presented various nonNewtonian models, e.g., Elabassbeshy et al. [9], Nadeem et al. [10], Lukaszewicz [11] and Kishan and Shashidar [12]. The study of boundary layer MHD flow towards a shrinking/stretching sheet has gained considerable attention of many researchers because of its frequent occurrence in industrial technology, geothermal application and high temperature plasmas applicable to nuclear fusion energy conversion and MHD power generation systems. Muhamin and Khamis [13] studied the effects of heat and mass transfer on the non-linear MHD viscous fluid flow over a shrinking sheet in the presence of suction. Mostafa A.A. Mahmaud [14] studied the non-uniform heat generation effect on heat transfer of a non-Newtonian powerlaw fluid over a non-linear stretching sheet. MHD mixed convection stagnation-point flow of a power-law non-Newtonian nanofluid towards a stretching surface with radiation and heat source/sink was analyzed by Madhu and Kishan [15]. Heat and mass transfer with hydrodynamic slip over a moving plate in porous media was investigated by Hamad et al. [16] via the Runge-Kutta-Fehlberg fourth-fifth order method. Over the years, considerable amount of literature has addressed the problem of fluid flow and heat transfer past a flat plate, especially in Newtonian fluids and to a limited extent in power-law fluids. Olajuwon [17] studied the convection heat and mass transfer in a power-law fluid over a flat plate in the presence of adverse pressure gradient. Kishan and Shashidar [18] studied MHD effects on a non-Newtonian power-law fluid past a continuously moving porous flat plate.

In the above investigations, Soret and Dufour's effects were neglected. However, when heat and mass transfer occurs simultaneously in a moving fluid the relations between the fluxes and driving potentials are of a more intricate nature. It has been found that an energy flux can be generated not only by a temperature gradient but also by a concentration gradient. The Soret effect, for instance, has been utilized for isotope separation and in mixtures between gases with very light molecular weight $\left(\mathrm{H}_{2}, \mathrm{He}\right)$. For medium molecular weight $\left(N_{2}\right.$, air) the Dufour effect was found to be of considerable magnitude such that it cannot be neglected. Postelnicu [19] used an implicit finite difference scheme to investigate the influence of a magnetic field on heat and mass transfer by natural convection from vertical surfaces in a porous media considering the Soret and Dufour effects. Rashidi et al. [20] discussed heat and mass transfer for MHD viscoelastic fluid 
flow over a vertical stretching sheet with considering the Soret and Dufour effects. The Soret and Dufour effects on MHD convective heat and mass transfer of a power-law fluid over an inclined plate with variable thermal conductivity in a porous medium were studied by Pal and Chatterjee [21].

In the above stated studies, no-slip thermal and solutal boundary conditions are considered. But, there might be a natural situation where no-slip boundary condition may not be applicable. In such circumstances, we may be forced to consider the slip boundary condition. Therefore, this study tries to fulfill this gap. Ibrahim and Shanker [22] investigated MHD boundary layer flow and heat transfer of a nanofluid past a permeable stretching sheet with velocity, thermal and solutal slip boundary conditions. Recently, Hirschhorn et al. [23] studied MDH boundary layer slip flow and heat transfer of a power-law fluid over a flat plate. This paper extends earlier works by examining the Soret and Dufour effects on MHD boundary layer heat and mass transfer of a power-law fluid over a flat plate with velocity, thermal and solutal slip boundary conditions.

\section{Mathematical formulation}

Consider a steady, laminar two-dimensional heat and mass transfer flow of an incompressible electrically conducting, viscous fluid obeying power-law model over a flat plate in the presence of transverse magnetic field $B$. The $x$-axis is taken along the direction of the flow and the $y$-axis normal to it. The thermophysical properties of the sheet and the fluid are assumed to be constant except for the viscosity of the power-law fluid which depends on the shear rate. We assume that the Dufour effect may be described by a second-order concentration derivative with respect to the transverse coordinate in the energy equation whereas the Soret effect is described by second-order temperature derivative in the mass-diffusion equation. With these assumptions and invoking the boundary layer approximations, the governing equations for the boundary layer flow, heat and mass transfer are

$$
\begin{aligned}
& \frac{\partial u}{\partial x}+\frac{\partial u}{\partial y}=0, \\
& u \frac{\partial u}{\partial x}+v \frac{\partial u}{\partial y}=v \frac{\partial}{\partial y}\left(\left|\frac{\partial u}{\partial y}\right|^{n-1} \frac{\partial u}{\partial y}\right)-\frac{\sigma B^{2}}{\rho}\left(u-U_{\infty}\right), \\
& u \frac{\partial T}{\partial x}+v \frac{\partial T}{\partial y}=\alpha \frac{\partial^{2} T}{\partial y^{2}}+\frac{D_{m} k_{T}}{c_{s} c_{p}} \frac{\partial^{2} C}{\partial y^{2}}, \\
& u \frac{\partial C}{\partial x}+v \frac{\partial C}{\partial y}=D_{m} \frac{\partial^{2} C}{\partial y^{2}}+\frac{D_{m} k_{T}}{T_{m}} \frac{\partial^{2} T}{\partial y^{2}} .
\end{aligned}
$$

Subject to the boundary conditions

$$
\begin{aligned}
& u=L_{I}\left(\frac{\partial u}{\partial y}\right), \quad v=0, \quad T=T_{w}+D_{I}\left(\frac{\partial T}{\partial y}\right), \quad C=C_{w}+P_{I}\left(\frac{\partial C}{\partial y}\right) \quad \text { at } \quad y=0, \\
& u \rightarrow U_{\infty}, \quad T \rightarrow T_{\infty}, \quad C \rightarrow C_{\infty} \quad \text { as } \quad y \rightarrow \infty
\end{aligned}
$$


where $u$ and $v$ are the velocity components in the directions of the $x$ and $y$ axes respectively, $\rho$ is the fluid density, $\sigma$ is the constant electrical conductivity of the fluid, $B$ is the magnetic field strength, $v=\frac{K}{\rho}$ is the kinematic viscosity, $K$ is the consistency coefficient, $U_{\infty}$ is the free stream velocity, $T$ is temperature, $\alpha=\frac{k}{\rho c_{p}}$ is the thermal diffusivity, $k$ is the thermal conductivity, $c_{p}$ is the specific heat capacity of the fluid, $c_{s}$ is the concentration susceptibility, $k_{T}$ is the thermal diffusion ratio, $D_{m}$ is the coefficient of mass diffusivity.

Also, $L_{l}=L \sqrt{\operatorname{Re}_{x}}$ is the velocity slip factor with $L$ being the initial value at the leading edge, $D_{l}=D \sqrt{\operatorname{Re}_{x}}$ is the thermal slip factor with $D$ being the initial value at the leading edge and $P_{l}=P \sqrt{\operatorname{Re}_{x}}$ is the concentration slip factor with $P$ being the initial value at the leading edge. Here, $T_{w}$ and $C_{w}$ are the temperature and concentration of the flat plate, $T_{\infty}$ and $C_{\infty}$ are the free stream temperature and concentration, and $\operatorname{Re}_{x}=\frac{U_{\infty}^{2-n} x^{n} \rho}{K}$ is the local Reynolds number.

The momentum, energy and mass equations can be transformed to a non-linear boundary value problem involving a system of coupled ordinary differential equations. In particular, we introduce the dimensionless similarity variables used by Reddy et al. [24] and defined as

$$
\begin{aligned}
& \Psi=b U_{\infty}\left(\frac{\frac{x}{b}}{\operatorname{Re}}\right)^{\frac{1}{n+1}} f(\eta), \\
& \eta=\left(\frac{\operatorname{Re}}{\frac{x}{b}}\right)^{\frac{1}{n+1}} \frac{y}{b}, \\
& T=T_{\infty}+\left(T_{w}-T_{\infty}\right) \theta(\eta), \\
& C=C_{\infty}+\left(C_{w}-T_{\infty}\right) \varnothing(\eta)
\end{aligned}
$$

where $\eta$ is the similarity variable, $\Psi$ is the stream function, $f, \theta$ and $\varnothing$ are the dimensionless similarity function, temperature and concentration respectively. Here $b$ is the characteristic length.

The velocity components $u$ and $v$ in terms of the stream function $\Psi(x, y)$ are given by

$$
u=\frac{\partial \Psi}{\partial y}, \quad v=-\frac{\partial \Psi}{\partial x}
$$

The generalized Reynolds number Re is defined by $\operatorname{Re}=\frac{\rho U_{\infty}^{2-n} b^{n}}{K}$.

Introducing the similarity transformations (2.6) and (2.7), the continuity equation is satisfied whereas the momentum, energy and mass equation given by Eqs (2.2), (2.3) and (2.4) are transformed the coupled non-linear ordinary differential equations of the form 


$$
\begin{aligned}
& n\left|f^{\prime \prime}\right|^{n-1} f^{\prime \prime \prime}+\frac{1}{n+1} f f^{\prime \prime}-M\left(f^{\prime}-1\right)=0, \\
& \theta^{\prime \prime}+\frac{1}{n+1} \operatorname{Pr} f \theta^{\prime}+\text { Du. } \varnothing^{\prime \prime}=0, \\
& \frac{1}{\mathrm{Le}} \varnothing^{\prime \prime}+\frac{1}{n+1} \operatorname{Pr} f \varnothing^{\prime}+\operatorname{Sr} \cdot \theta^{\prime \prime}=0 .
\end{aligned}
$$

Here primes denote differentiation with respect to $\eta$, and where $\quad M=\frac{\sigma B^{2} x}{\rho U_{\infty}}$ is the magnetic parameter, $\operatorname{Pr}=\frac{U x}{\alpha} \operatorname{Re}_{x}^{\frac{-2}{n+1}}$ is the Prandtl number, $\mathrm{Le}=\frac{\alpha}{D_{m}}$ is the Lewis number, $\mathrm{Du}=\frac{D_{m} k_{T}}{c_{s} c_{p}} \cdot \frac{\left(C_{w}-C_{\infty}\right)}{\left(T_{w}-T_{\infty}\right) \alpha}$ is the Dufour number, $\mathrm{Sr}=\frac{D_{m} k_{T}}{T_{m \alpha}} \cdot \frac{\left(T_{w}-T_{\infty}\right)}{\left(C_{w}-C_{\infty}\right)}$ is the Soret number.

Boundary conditions (2.5a) and (2.5b) are transformed into

$$
\begin{aligned}
& f(\eta)=0, \quad f^{\prime}(\eta)=A_{1} f^{\prime \prime}(\eta), \quad \theta(\eta)=1+B_{l} \theta^{\prime}(\eta), \quad \varnothing(\eta)=1+C_{l} \varnothing^{\prime}(\eta) \text { at } \eta=0, \\
& f^{\prime}(\eta) \rightarrow 1, \quad \theta(\eta) \rightarrow 0, \quad \varnothing(\eta) \rightarrow 0 \quad \text { as } \quad \eta \rightarrow \infty
\end{aligned}
$$

where $A_{l}, B_{l}$ and $C_{l}$ are, respectively, the velocity, temperature and concentration slip parameters, which are further defined as

$$
\begin{aligned}
& A_{I}=L \frac{U_{\infty} \rho}{K}, \\
& B_{I}=D \frac{U_{\infty} \rho}{K}, \\
& C_{l}=P \frac{U_{\infty} \rho}{K} .
\end{aligned}
$$

In practical applications, the physical quantities of principal interest are the skin-friction coefficient $C_{f}$, local Nusselt number $\mathrm{Nu}_{x}$ and local Sherwood number $\mathrm{Sh}_{x}$ which indicate the physical wall shear stress, rate of heat transfer and the rate of mass transfer, respectively. These physical quantities are defined respectively as

$$
C_{f}=\frac{2 \tau_{w}}{\rho U_{\infty}^{2}}=2\left[f^{\prime \prime}(0)\right]^{n} \operatorname{Re}_{x}^{1 / n+1}, \quad \mathrm{Nu}_{x}=\frac{q_{w} x}{k\left(T_{w}-T_{\infty}\right)}=-\theta^{\prime}(0) \operatorname{Re}_{x}^{1 / n+1},
$$


and

$$
\operatorname{Sh}_{x}=\frac{J_{w} x}{D_{m}\left(C_{w}-C_{\infty}\right)}=-\varnothing^{\prime}(0) \operatorname{Re}_{x}^{1 / n+1}
$$

where the wall shear stress $\tau_{\mathrm{w}}$, the heat flux at the wall $q_{w}$ and the mass flux at the wall $J_{w}$ are defined as

$$
\tau_{w}=\mu_{0}\left[\frac{\partial u}{\partial y}\right]_{y=0}, \quad q_{w}=-k\left[\frac{\partial T}{\partial y}\right]_{y=0} \quad \text { and } \quad J_{w}=-D_{m}\left[\frac{\partial C}{\partial y}\right]_{y=0} .
$$

\section{Numerical procedure}

The combined effects of various physical parameters will have a great impact on heat and mass transfer characteristics. The non-linearity of the basic equations and additional mathematical difficulties associated with the solution part has led us to use a numerical method. In this section, an efficient implicit finite difference scheme along with quasi-linearization technique have been employed to analyze the flow model for the above coupled ordinary differential Eqs (2.9), (2.10) and (2.11) along with the boundary conditions (2.12) for different values of the governing parameters. The transformed non-linear differential Eq.(2.9) is first linearized by quasi-linearization technique discussed by Bellman and Kalaba [25]. Now by applying the implicit finite difference scheme, these equations are transformed to system of linear equations. To carry out the computational procedure, first the momentum equation is solved which gives the values of $f$ necessary for obtaining the solution of coupled energy and concentration equations under the boundary conditions (2.12) by the Gauss Seidal iteration procedure. The numerical solutions of $f$ are considered as $(\mathrm{n}+1)^{\text {th }}$ order iterative solutions and $\mathrm{F}$ are the $n^{\text {th }}$ order iterative solutions. To prove convergence of the finite difference scheme, the computation is carried out for slightly changed value of $h$ by running same program. No significant change was observed in the value. At every position, the iteration process continues until the convergence criterion for all the variables, $10^{-5}$ is achieved.

\section{Results and discussions}

Numerical computations are carried out for several sets of values of the governing parameters, namely, the magnetic parameter $M$, power-law index $n$, Prandtl number Pr, Lewis number Le, velocity slip parameter $A_{l}$, temperature slip parameter $B_{l}$, concentration slip parameter $C_{l}$, Soret number $\mathrm{Sr}$ and Dufour number Du. In order to get a clear insight into the physical problem, numerical results are displayed with the help of graphical illustrations. Graphical illustration of the results is very useful and practical to discuss the effect of different parameters.

To validate our results, the numerical computations of skin friction coefficients, Nusselt number and Sherwood number which are respectively proportional to $f^{\prime \prime}(0),-\theta^{\prime}(0)$ and $-\varnothing^{\prime}(0)$. are presented in a tabular form. Tables $1-5$ show the effect of the power-law index $n$, velocity slip parameter $A_{l}$, temperature slip parameter $B_{l}$, concentration slip parameter $C_{l}$, Soret number $\mathrm{Sr}$ and Dufour number $\mathrm{Du}$ on the coefficient of skin friction $f^{\prime \prime}(0)$, Nusselt number $-\theta^{\prime}(0)$ and Sherwood number $-\varnothing^{\prime}(0)$ respectively. It can be seen that the effect of the power-law index $n$ is to decrease the skin friction coefficient, Nusselt number and Sherwood number. The coefficient of skin friction, Nusselt number and Sherwood number decrease with the increase in the velocity slip parameter, temperature slip parameter and concentration slip parameter respectively. It is evident from Tabs 4 and 5 that an increase in the Soret number (or decrease in the Dufour number) decreases the Nusselt number but increases the Sherwood number. 
Table 1. Numerical results of the skin friction coefficient $f^{\prime \prime}(0)$ for different values of the velocity slip parameter $A_{1}$ with $M=0.2, \operatorname{Pr}=0.7, \mathrm{Le}=1.0, \mathrm{Sr}=0.04, \mathrm{Du}=0.02, B_{1}=0, C_{l}=0$.

\begin{tabular}{|c|c|c|c|}
\hline & \multicolumn{3}{|l|}{ Values of $f^{\prime \prime}(0)$} \\
\hline$A_{1}$ & $n=0.5$ & $n=1.0$ & $n=1.5$ \\
\hline 0 & 0.302737 & 0.136838 & 0.11551 \\
\hline 0.2 & 0.25936 & 0.116271 & 0.098584 \\
\hline 0.4 & 0.224204 & 0.100644 & 0.085674 \\
\hline 0.6 & 0.196388 & 0.088525 & 0.075609 \\
\hline
\end{tabular}

Table 2. Numerical results of the Nusselt number $-\theta^{\prime}(0)$ for different values of the temperature slip parameter $B_{1}$ with $M=0.2, \operatorname{Pr}=0.7, \mathrm{Le}=1.0, \mathrm{Sr}=0.04, \mathrm{Du}=0.02, A_{l}=0, C_{l}=0$.

\begin{tabular}{|c|c|c|c|}
\hline & \multicolumn{3}{|l|}{ Values of $-\theta^{\prime}(0)$} \\
\hline$B_{1}$ & $n=0.5$ & $n=1.0$ & $n=1.5$ \\
\hline 0 & 0.307352 & 0.258658 & 0.247737 \\
\hline 0.2 & 0.286032 & 0.244551 & 0.235126 \\
\hline 0.4 & 0.267646 & 0.231998 & 0.223833 \\
\hline 0.6 & 0.2516 & 0.220731 & 0.21361 \\
\hline
\end{tabular}

Table 3. Numerical results of the Sherwood number $-\varnothing^{\prime}(0)$ for different values of the concentration slip parameter $C_{l}$ with $M=0.2, \operatorname{Pr}=0.7, \mathrm{Le}=1.0, \mathrm{Sr}=0.04, \mathrm{Du}=0.02, A_{1}=0, C_{1}=0$.

\begin{tabular}{|c|c|c|c|}
\hline & \multicolumn{3}{|l|}{ Values of $-\varnothing^{\prime}(0)$} \\
\hline$C_{l}$ & $n=0.5$ & $n=1.0$ & $n=1.5$ \\
\hline 0 & 0.261925 & 0.236719 & 0.231336 \\
\hline 0.2 & 0.244299 & 0.223908 & 0.219543 \\
\hline 0.4 & 0.228995 & 0.212503 & 0.208896 \\
\hline 0.6 & 0.215569 & 0.202133 & 0.199188 \\
\hline
\end{tabular}

Table 4. Numerical results of the Nusslet number $-\theta^{\prime}(\theta)$ for different values of the Soret and Dufour number with $M=0.2, \operatorname{Pr}=0.7, \mathrm{Le}=1.0, A_{1}=0, B_{1}=0, C_{1}=0$.

\begin{tabular}{|c|c|c|c|}
\hline & \multicolumn{3}{|c|}{ Values of $-\theta^{\prime}(0)$} \\
\hline $\mathrm{Sr}, \mathrm{Du}$ & $n=0.5$ & $n=1.0$ & $n=1.5$ \\
\hline $0.08,0.01$ & 0.330075 & 0.269637 & 0.255924 \\
\hline $0.04,0.02$ & 0.307352 & 0.258658 & 0.247737 \\
\hline $0.02,0.04$ & 0.261925 & 0.236719 & 0.231336 \\
\hline $0.01,0.08$ & 0.171099 & 0.192867 & 0.198493 \\
\hline
\end{tabular}

Table 5. Numerical results of the Sherwood number $-\varnothing^{\prime}(0)$ for different values of the Soret and Dufour number with $M=0.2, \operatorname{Pr}=0.7, \mathrm{Le}=1.0, A_{1}=0, B_{1}=0, C_{1}=0$.

\begin{tabular}{|c|c|c|c|}
\hline & \multicolumn{3}{|c|}{ Values of $-\varnothing^{\prime}(0)$} \\
\hline $\mathrm{Sr}, \mathrm{Du}$ & $n=0.5$ & $n=1.0$ & $n=1.5$ \\
\hline $0.08,0.01$ & 0.171099 & 0.192867 & 0.198493 \\
\hline $0.04,0.02$ & 0.261925 & 0.236719 & 0.231336 \\
\hline $0.02,0.04$ & 0.307352 & 0.258658 & 0.247737 \\
\hline $0.01,0.08$ & 0.330075 & 0.269637 & 0.255924 \\
\hline
\end{tabular}


Figure 1 demonstrates the dimensionless velocity profile $f^{\prime}$ for different values of the magnetic field parameter $M$. The general trend is that $f^{\prime}$ increases monotonically as the space variable increases. The effect of increasing values of the magnetic field parameter results in flattering of velocity profile $f^{\prime}$ in all the cases. Hence it is very clear that the transverse magnetic field opposes the transport phenomena. This is due to the fact that the increase in the magnetic number leads to the enhancement of Lorentz force and hence Lorentz force produces more resistance to transport phenomena. Figure 2 display the distinction of velocity profile with respect to variation in the velocity slip parameter. On observing these figures, the velocity graph increases with the increase in the values of the velocity slip parameter $A_{1}$.

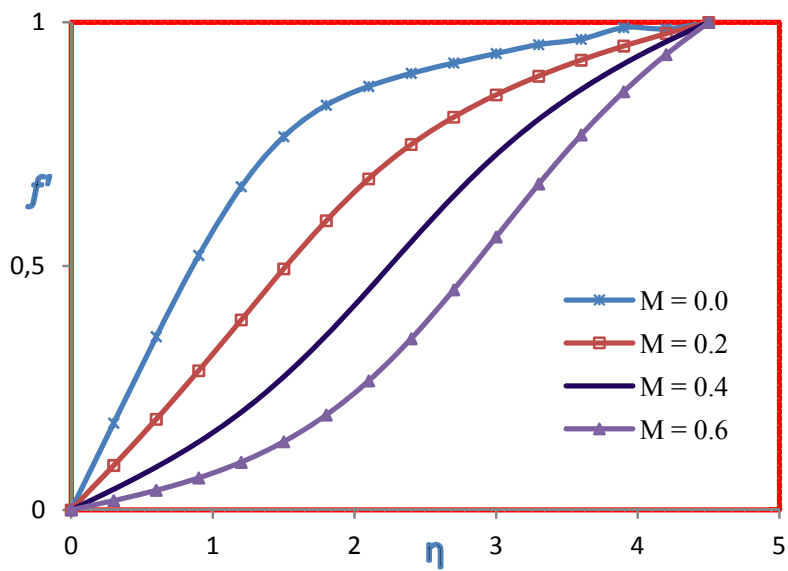

(a) Pseudo Plastic fluids $(n=0.5)$

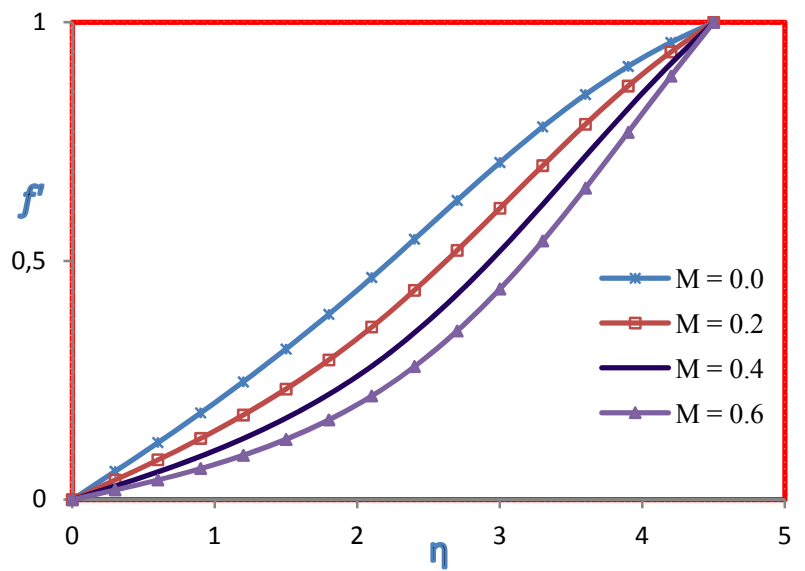

(b) Newtonian fluids $(n=1.0)$

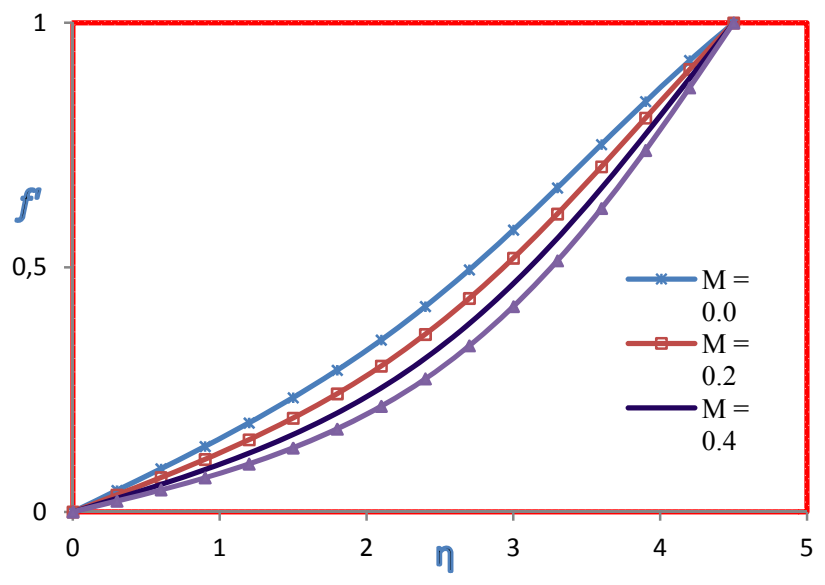

(c) Dilatant fluids $(n=1.5)$

Fig.1. Variation of velocity profiles for various values of magnetic field parameter $M$ with $\operatorname{Pr}=0.7$, $\mathrm{Le}=1.0, \mathrm{Sr}=0, \mathrm{Du}=0, A_{1}=0, B_{1}=0, C_{1}=0$. 


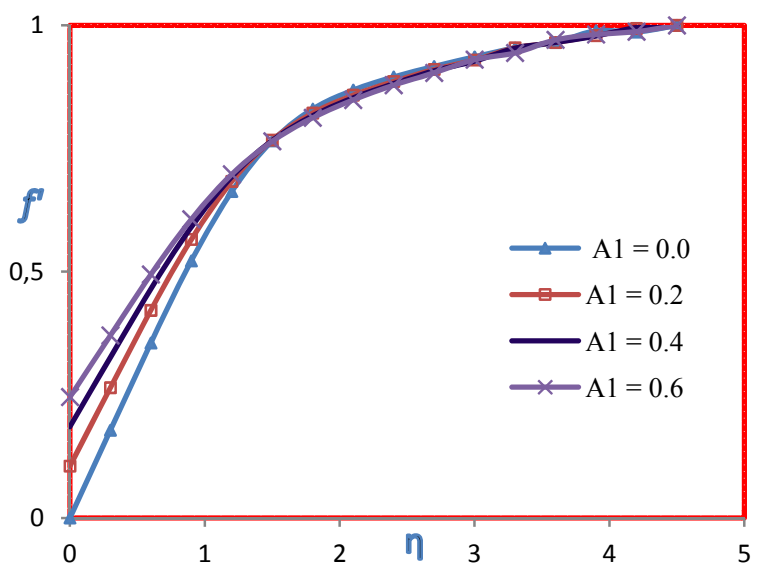

(a) Pseudo Plastic fluids $(n=0.5)$

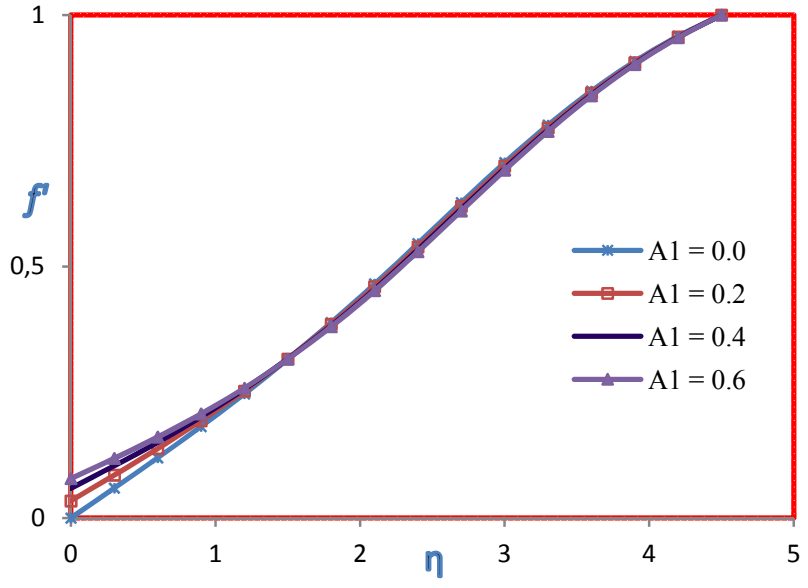

(b) Newtonian fluids $(n=1.0)$

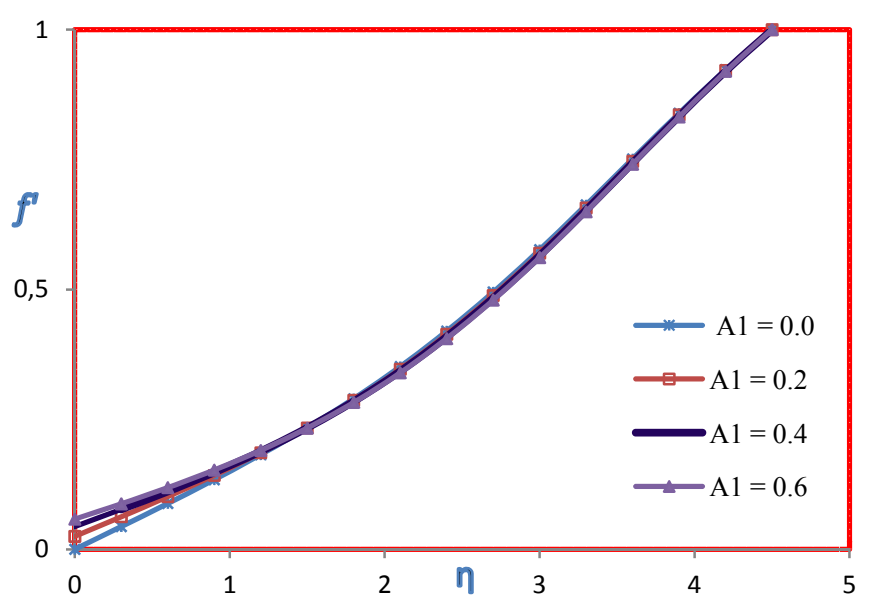

(c) Dilatant fluids $(n=1.5)$

Fig.2. Variation of velocity profiles for various values of velocity slip parameter $A_{1}$ with $\operatorname{Pr}=0.7, \mathrm{Le}=1.0$, $\mathrm{Sr}=0, \mathrm{Du}=0, M=0, B_{1}=0, C_{1}=0$.

The influence of the Prandtl number Pr on the heat transfer process in shown in Fig.3. These graphs reveal that the temperature decreases with an increase in the Prandtl number. This is because thermal boundary layer thickness decreases as the Prandtl number increases. Hence the rate of thermal diffusion is slow with an increase in the Prandtl number. The effect of thermal slip parameter $B_{l}$ on the temperature profiles is shown in Fig.4. It is noticed from the profiles that the wall temperature $\theta(0)$ and the thermal boundary layer thickness decreases with the increase in the thermal slip parameter. Figure 5 illustrates the variation of concentration in response to the change in the Lewis number Le. The graphs reveal that the concentration decreases with the increase in the Lewis number. This is probably due to the fact that the increase in Le decreases the mass diffusivity and hence the concentration boundary layer descends. Figure 6 demonstrates the variation of concentration profiles in response to the change in the concentration slip parameter $C_{l}$. It can be noticed from the graphs that by increasing $C_{l}$, the wall ccentration $\varphi(0)$ and concentration profiles decrease and hence concentration boundary layer thickness decreases. 


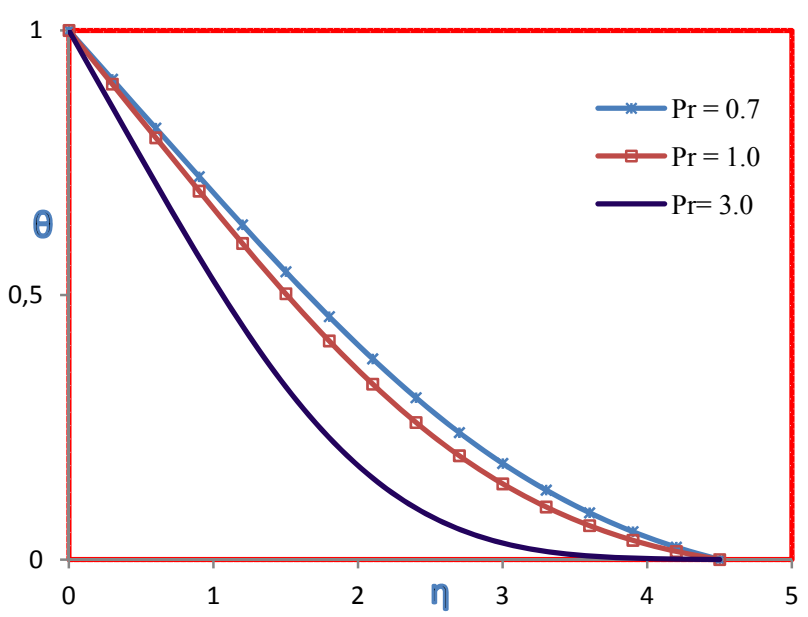

(a) Pseudo Plastic fluids $(n=0.5)$

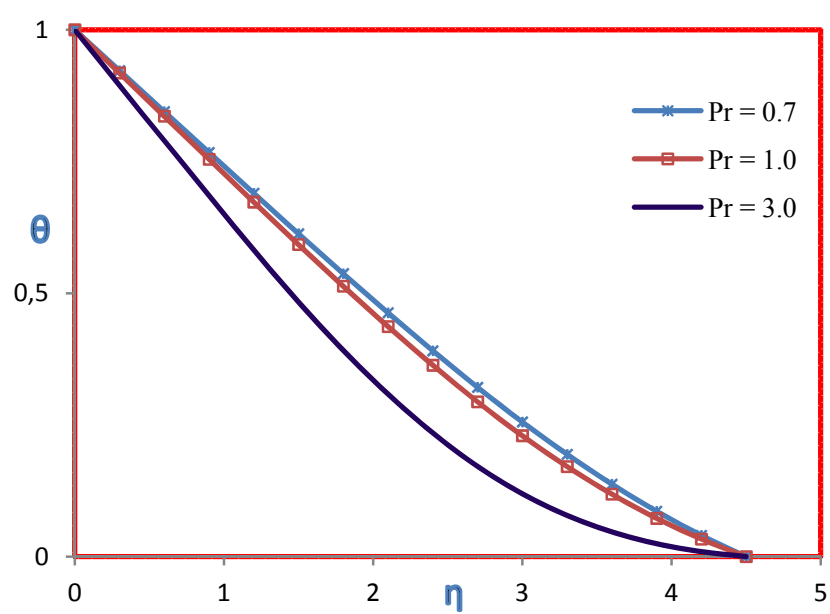

(b) Newtonian fluids $(n=1.0)$

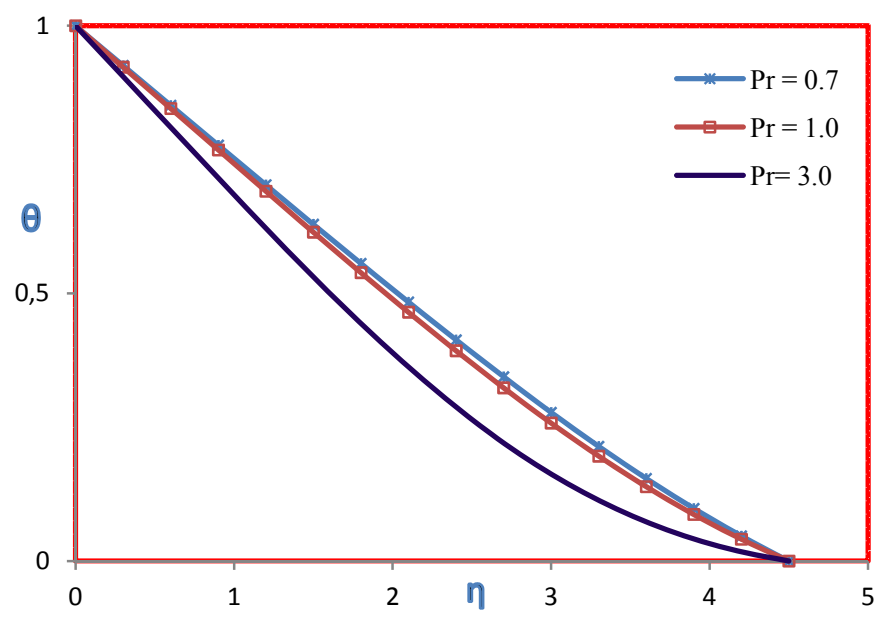

(c) Dilatant fluids $(n=1.5)$

Fig.3. Variation of temperature profiles for various values of Prandtl number $\operatorname{Pr}$ with $M=0.2, \mathrm{Le}=1.0$, $\mathrm{Sr}=0.04, \mathrm{Du}=0.02, A_{1}=0, B_{1}=0, C_{1}=0$.

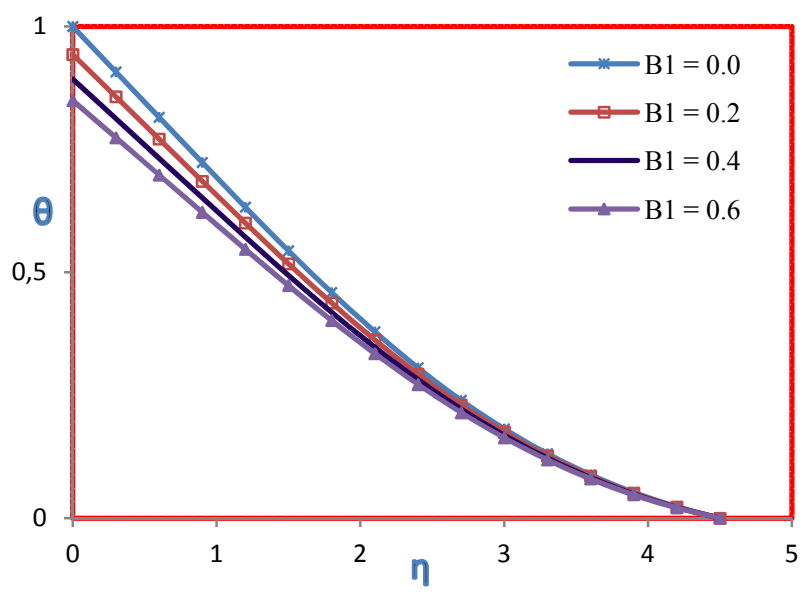

(a) Pseudo Plastic fluids $(n=0.5)$

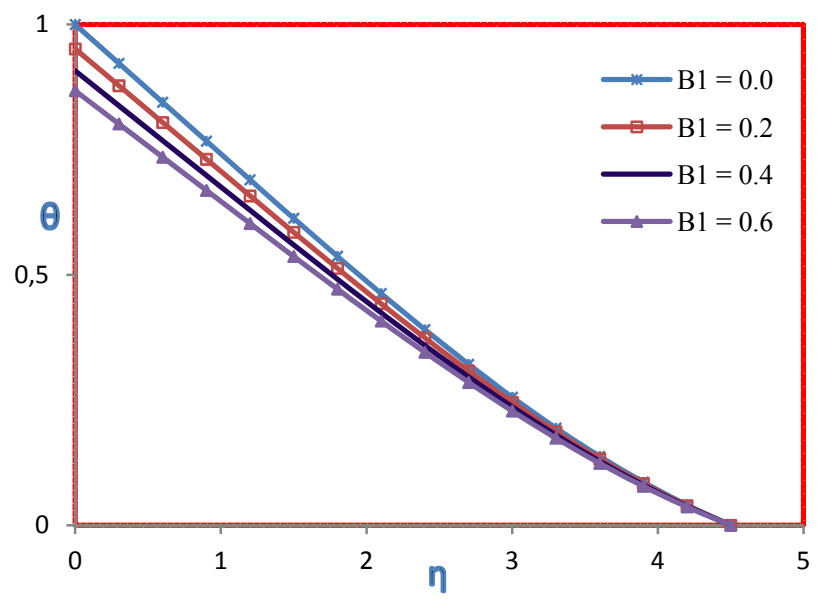

(b) Dilatant fluids $(n=1.0)$ 


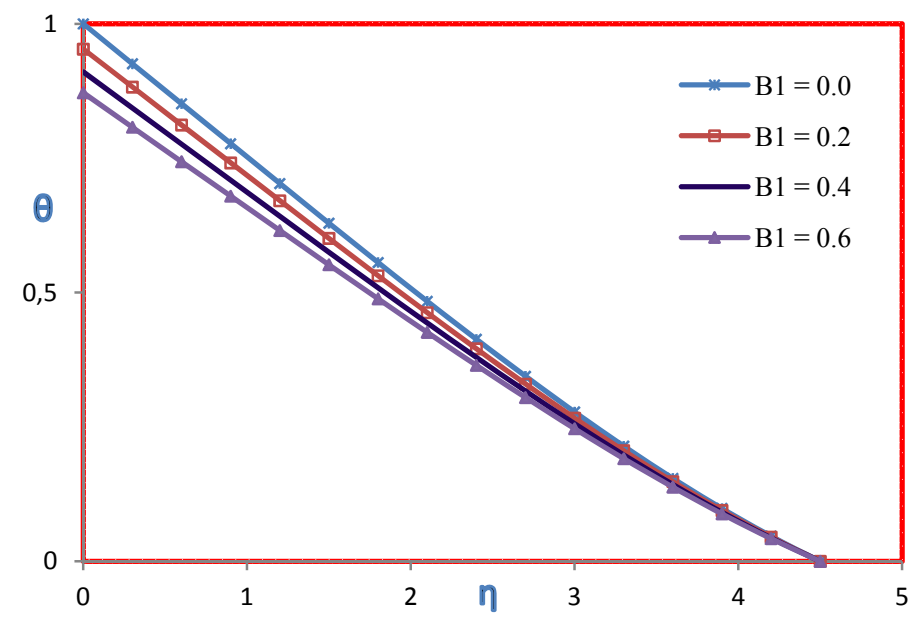

(c) Dilatant fluids ( $n=1.5$ )

Fig.4. Variation of temperature profiles for various values of temperature slip parameter $B_{1}$ with $M=0.2$, $\operatorname{Pr}=0.7, \mathrm{Le}=1.0, \mathrm{Sr}=0.04, \mathrm{Du}=0.02, A_{1}=0, C_{1}=0$.

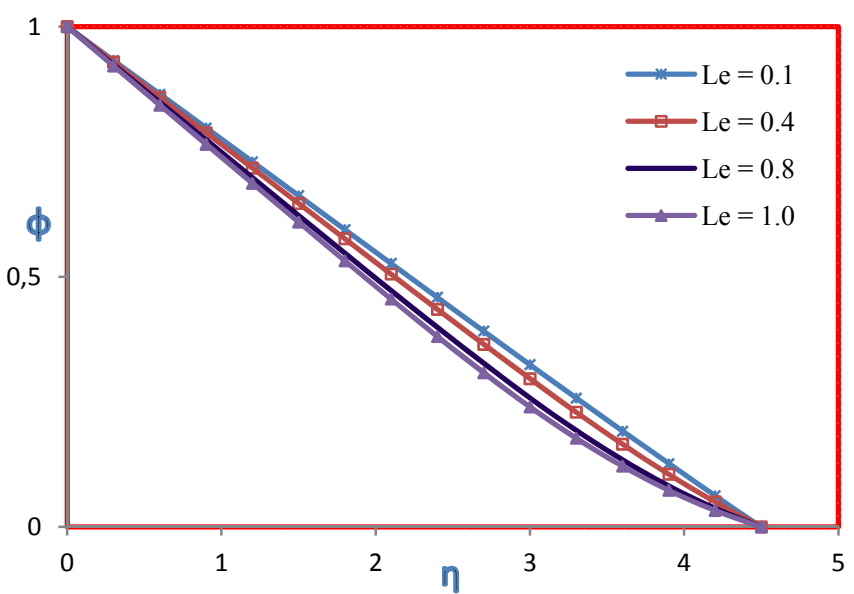

(a) Pseudo plastic fluids $(n=0.5)$

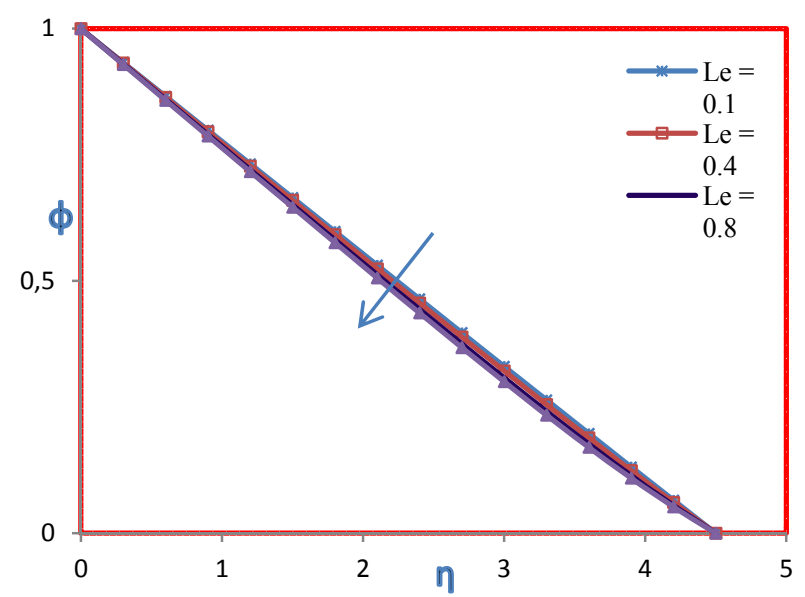

(b) Newtonian fluids $(n=1.0)$

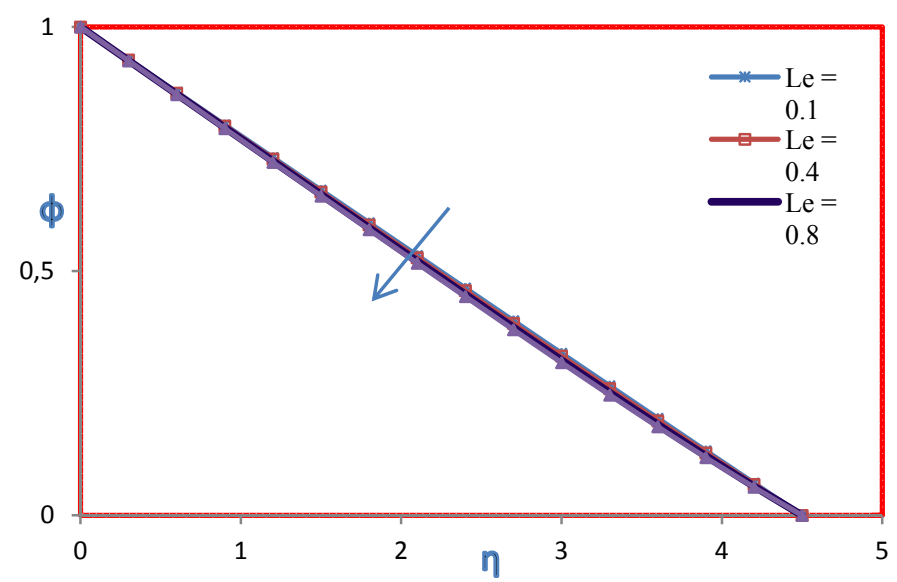

(c) Dilatant fluids $(n=1.5)$

Fig.5. Variation of concentration profiles for various values of Lewis number Le with $M=0.2, \operatorname{Pr}=0.7$, $\mathrm{Sr}=0.04, \mathrm{Du}=0.02, A_{1}=0, B_{I}=0, C_{1}=0$. 


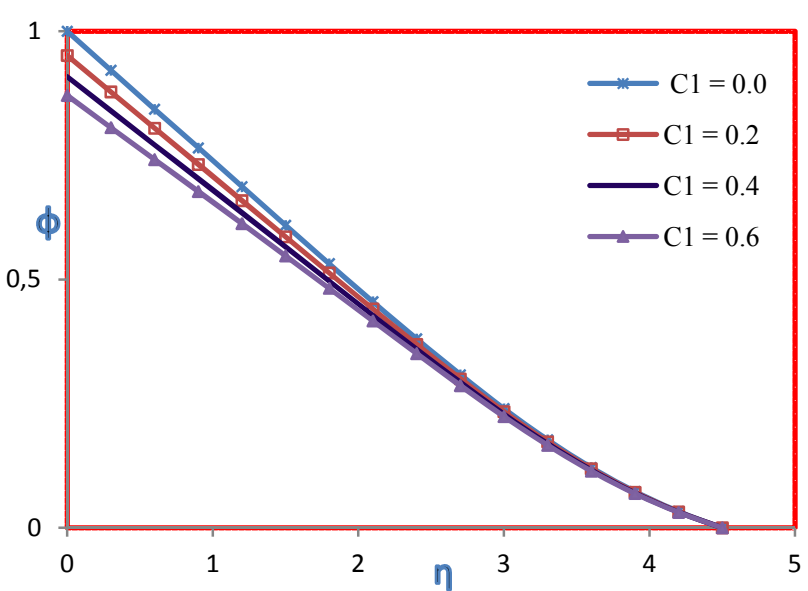

(a) Pseudo plastic fluids $(n=0.5)$

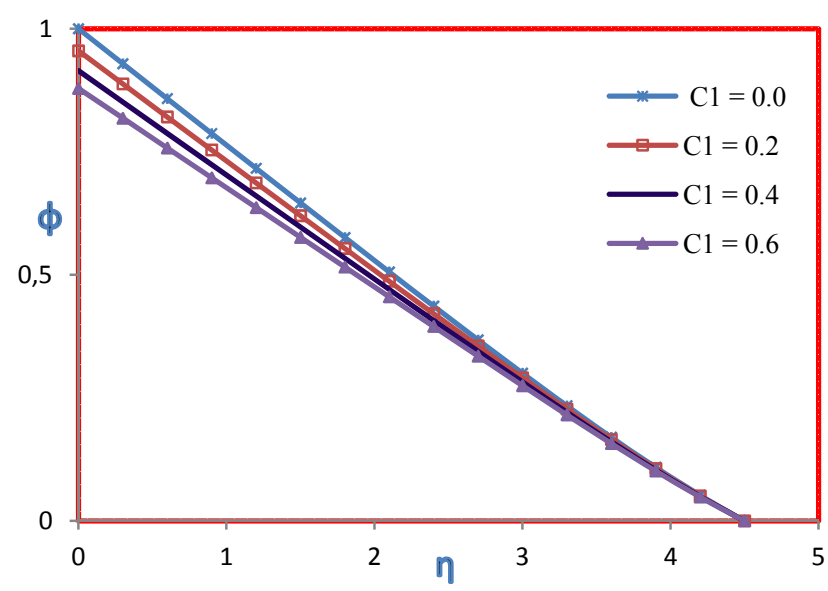

(b) Newtonian fluids $(n=1.0)$

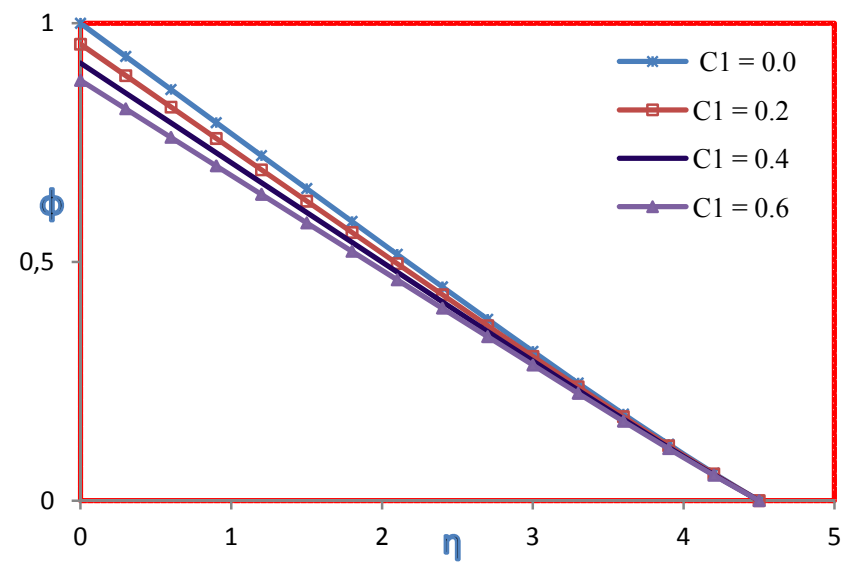

(c) Dilatant fluids $(n=1.5)$

Fig.6. Variation of concentration profiles for various values of concentration slip parameter $C_{1}$ with $M=0.2, \operatorname{Pr}=0.7, \mathrm{Le}=1.0, \mathrm{Sr}=0.04, \mathrm{Du}=0.02, A=0, B=0$.

Figure 7 displays the variation of velocity, temperature and concentration profiles with the powerlaw index $n$. It is evident from the figures that the velocity profiles decrease with an increase in the powerlaw index whereas the temperature and concentration profiles increase with an increase in the power-law index. The variations of the Soret and Dufour number on the temperature and concentration fields are displayed respectively in Figs 8 and 9. The mass flux due to the temperature gradient is defined as the Soret effect whereas enthalpy flux due to concentration gradient - the Dufour effect. These graphs reveal that a decrease in the Soret number (or increase in the Dufour number) enhances the temperature profiles and reduces the concentration profiles. It is due to the fact that an increase in the Soret number cools down the fluid and hence the temperature reduces. 


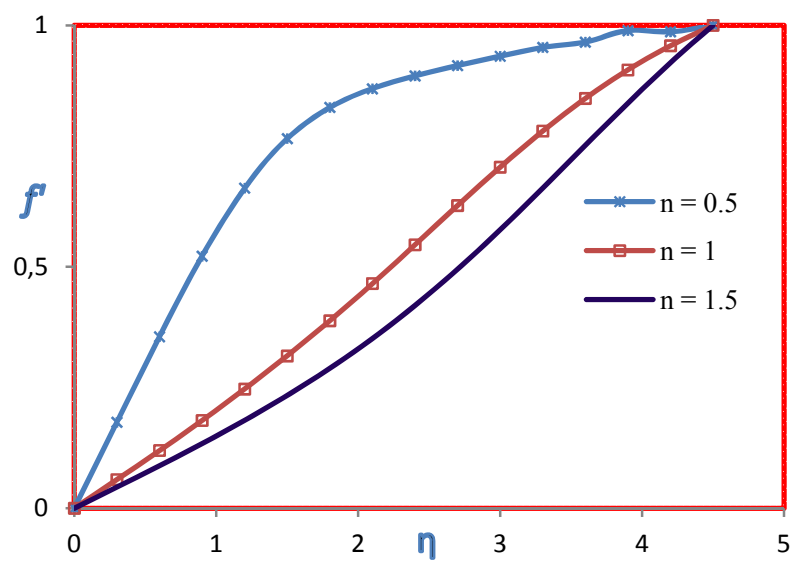

(a) Velocity profiles

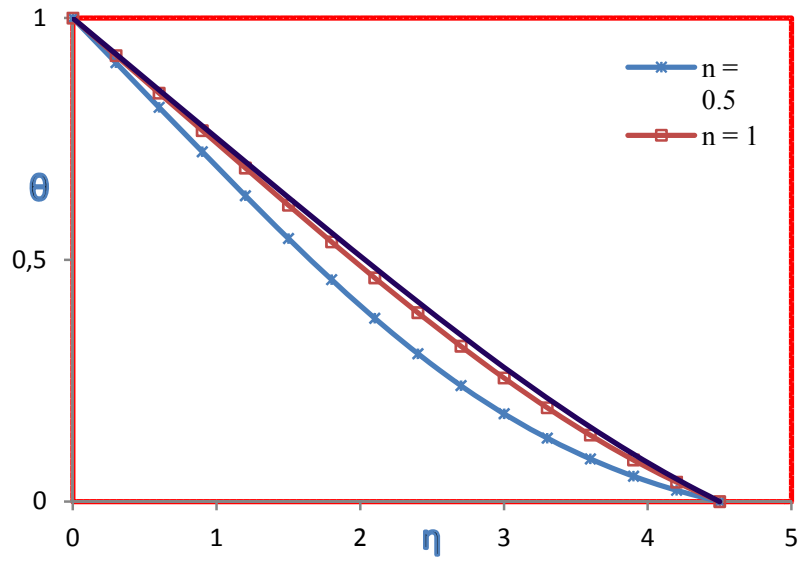

(b) Temperature profiles

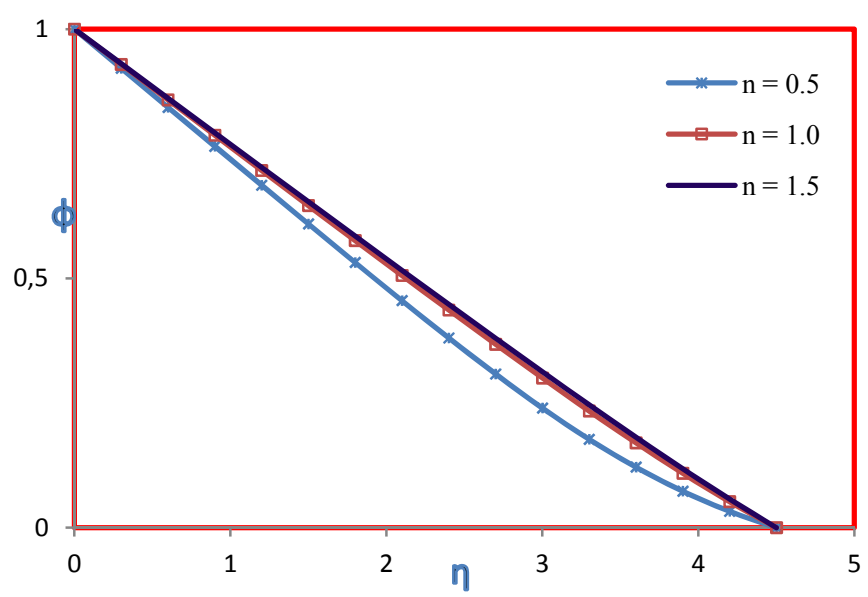

(c) Concentration profiles

Fig.7. Variation of velocity, temperature and concentration profiles for various values of power-law index $n$ with $M=0.2, \mathrm{Pr}=0.7, \mathrm{Le}=1.0, \mathrm{Sr}=0.04, \mathrm{Du}=0.02, A_{1}=0, B_{1}=0, C_{l}=0$.

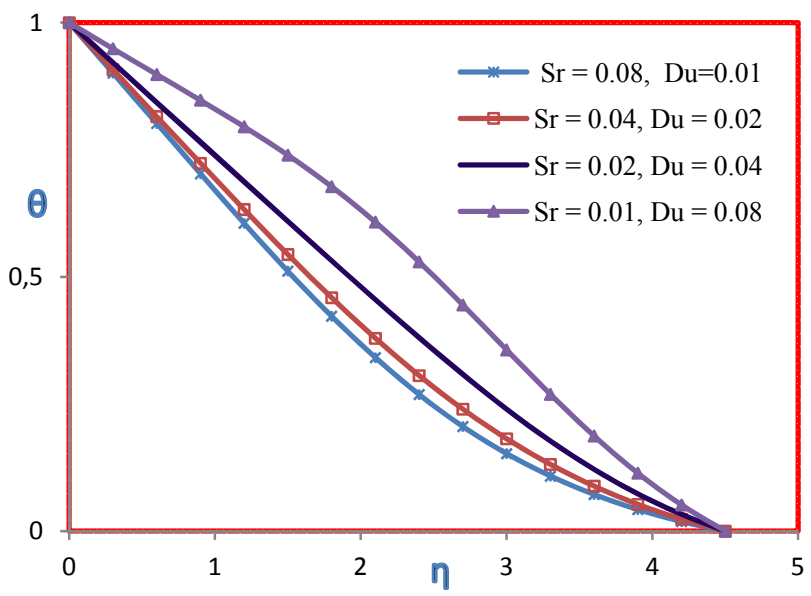

(a) Pseudo plastic fluids $(n=0.5)$

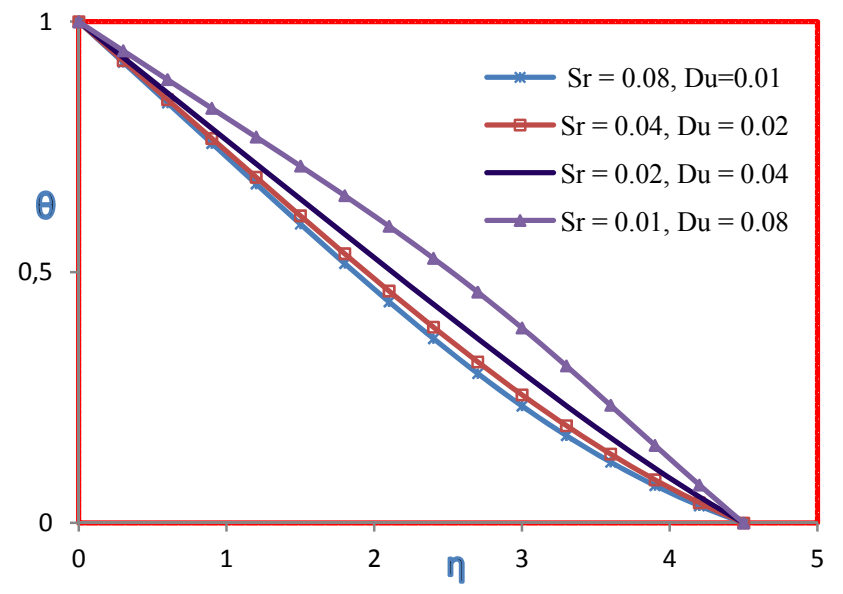

(b) Newtonian fluids $(n=1.0)$ 


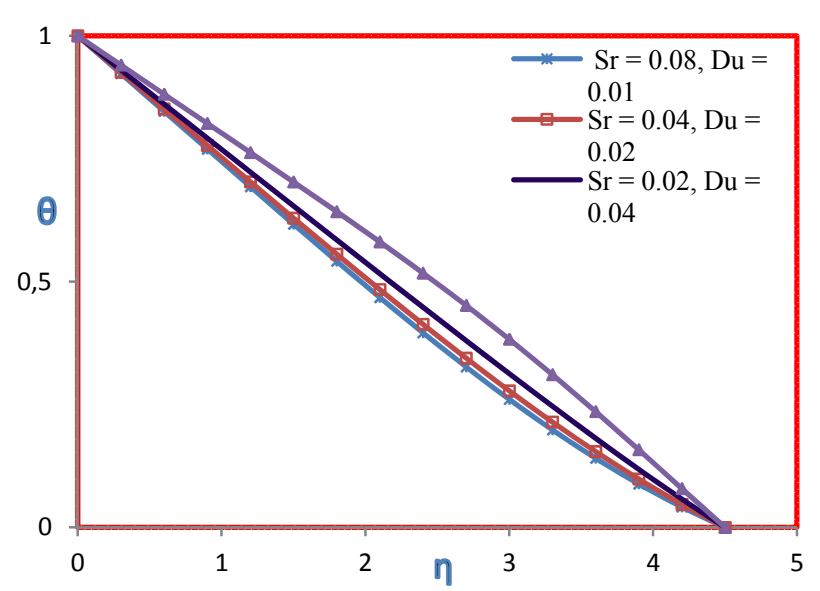

(c) Dilatant fluids $(n=1.5)$

Fig.8. Variation of temperature profiles for various values of Soret number and Dufour number with $M=0.2, \operatorname{Pr}=0.7, \mathrm{Le}=1.0, A_{1}=0, B_{1}=0, C_{l}=0$.

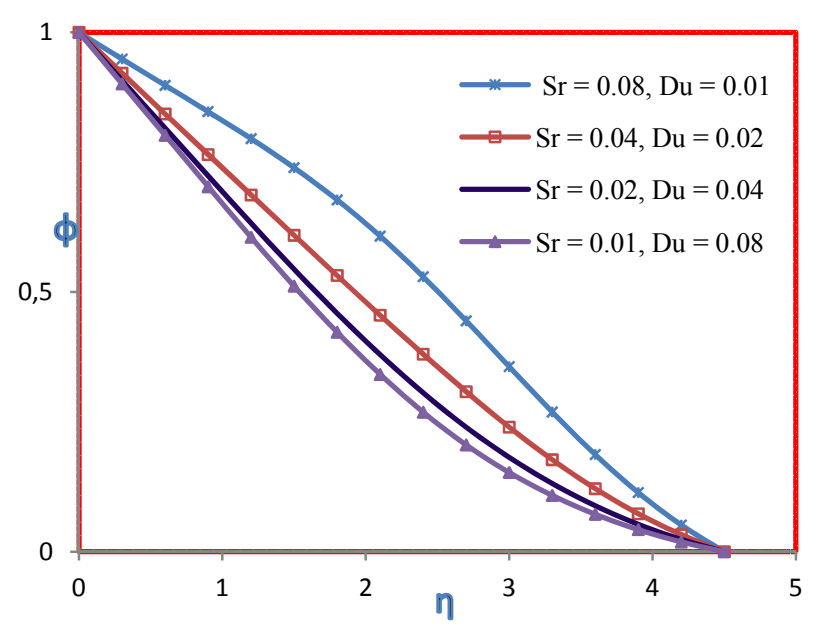

(a) Pseudo plastic fluids $(n=0.5)$

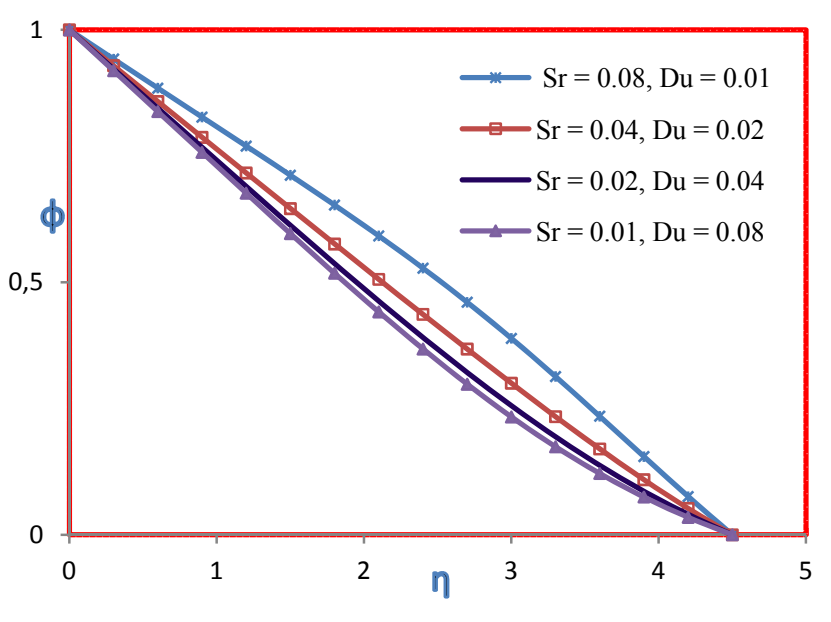

(b) Newtonian fluids $(n=1.0)$

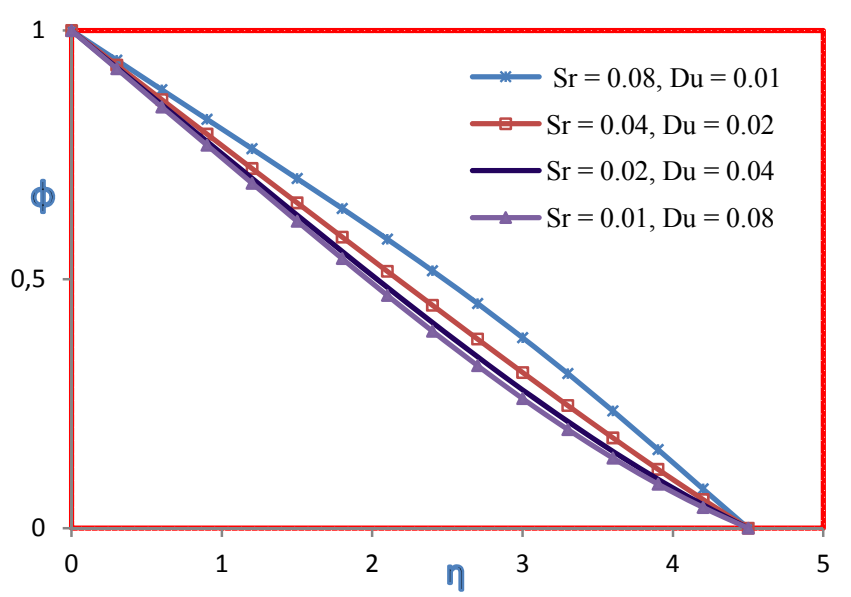

(c) Dilatant fluids $(n=1.5)$

Fig.9. Variation of concentration profiles for various values of Soret number and Dufour number with $M=0.2, \operatorname{Pr}=0.7, \mathrm{Le}=1.0, A_{l}=0, B_{1}=0, C_{l}=0$. 


\section{Conclusions}

The present study describes the heat and mass transfer of a power-law fluid flow over a flat plate in the presence of a transverse magnetic field by taking into account the Soret and Dufour effects. From the above investigation, the following conclusions may be drawn:

1. The higher the velocity, temperature and concentration slip parameters, the lower the coefficient of skin friction, Nusselt number and Sherwood number, respectively.

2. A high thermal diffusion (Soret number) effect enhances the rate of heat transfer and reduces the rate of mass transfer.

3. Velocity reduces with the high magnetic field effect.

4. Velocity at the surface of the plate decreases with the increase in the velocity slip parameter.

5. The thickness of the boundary layer decreases with the increase in the Prandtl number and the temperature slip parameter.

6. Concentration boundary layer thickness decreases with the increase in the Lewis number and the concentration slip parameter.

7. The effect of the Soret number is to reduce the temperature and enhance the concentration.

\section{Nomenclature}

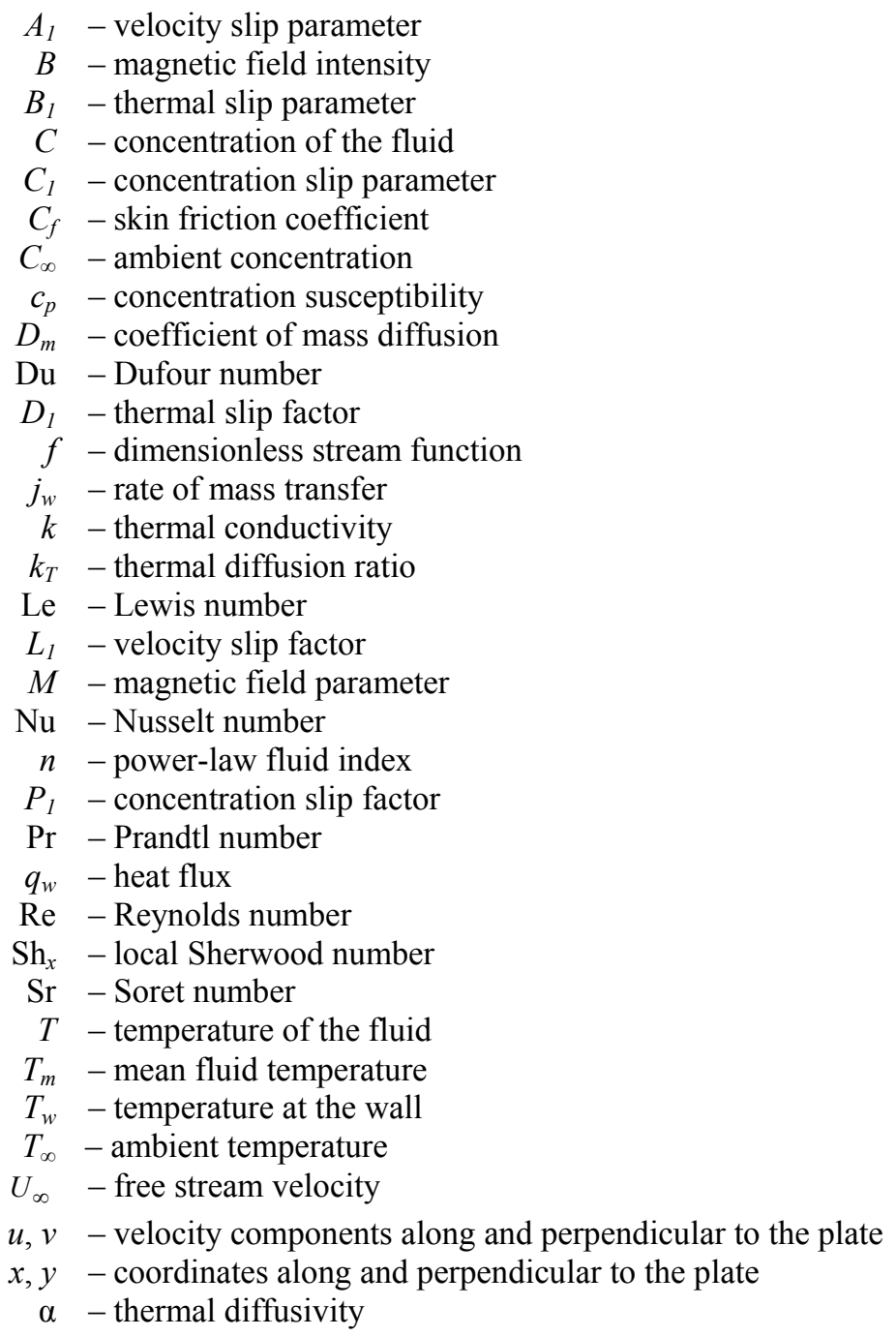


$\eta$ - dimensionless similarity variable

$\theta$ - dimensionless temperature

$\mu-$ magnetic permeability

$\mu_{0}-$ dynamic coefficient of viscosity

$v-$ kinematic viscosity

$\rho$ - density

$\sigma \quad$ - electrical conductivity

$\tau$ - tensor of stress

$\phi$ - dimensionless concentration

$\psi-$ stream function

\section{References}

[1] Sakiadas B.C. (1961): Boundary layer behavior on continuous solid surfaces. I. Boundary layer equations for two dimensional axisymmatric flow. - AIChEJ, vol.7, pp.26-28.

[2] Crane L.J. (1970): Flow past a stretching plane. - Z. Angew. Math. Physics, vol.21, pp.645-647.

[3] Anjali Devi S.P. and Thyagarajan M. (2006): Steady non-linear hydromagnetic flow and heat transfer over a stretching surface of variable temperature. - Heat Mass Transfer, vol.42, pp.671-677.

[4] Chakrabarti A. and Gupta A.S. (1979): Hydromagnetic flow and heat transfer over a stretching sheet. - Q. Appl. Math., vol.37, pp.73-78.

[5] Chen C.K. (1988): Laminar mixed convection adjacent to vertical continuously stretching sheets. - Heat Mass Transfer, vol.33, pp.471-476.

[6] Blassius (1908): Grenzschichten in Flussigkeiten mit kleiner Reibung. - Z. Math. U. Physics, vol.56, pp.1-36.

[7] Abdul-Rahim, Khaled A. and Chamkha Ali J. (2001): Variable porosity and thermal dispersion effects on coupled heat and mass transfer by natural convection from a surface embedded in a non-metallic porous medium. International Journal of Numerical Methods for Heat and Fluid Flow, vol.11, No.5, pp.413-419.

[8] Pop I. and Takhar H. (1983): Thermal convection near a partly insulated vertical flat plate embedded in a saturated porous medium. - Mechanics Research Communications, vol.10, No.2, pp.83-89.

[9] Elabashbeshy E.M., Emam T.G. and Abdel-Wahed M.S. (2011): Three dimensional flow over a stretching surface with thermal radiation and heat generation in the presence of chemical reaction and suction/injection. International Journal of Energy Technology, vol.16, pp.1-8.

[10] Nadeem S., Hussian A. and Khan M. (2010): Ham solutions for boundary layer flow in the region of the stagnation point towards a stretching sheet. - Communications in Nonlinear Science and Numerical Simulation, vol.15, No.3, pp.475-481.

[11] Lukaszewicz G. (2003): Asymptotic behavior of micro-polar fluid flows. - International Journal of Engineering Science, vol.41, No.3, pp.259-69.

[12] Kishan N. and Shashidar Reddy B. (2011): Quasi-linearization approach to MHD effects on boundary layer flow of power-law fluids past a semi infinite plate with thermal dispersion. - International Journal of Non-Linear Science, vol.11, No.3, pp.301-311.

[13] Muhaimin R.K. and Khamis A.B. (2008): Effects of heat and mass transfer on non-linear MHD boundary layer flow over a shrinking sheet in the presence of suction. - Applied Mathematics and Mechanics (English Edition), vol.29, No.10, pp.1309-1317. DOI 10.1007/s10483-008-1006-z.

[14] Mostafa A.A., Mahmoud Ahmed and Megahed M. (2012): Non-uniform heat generation effect on heat transfer of a non-Newtonian power-law fluid over a non-linearly stretching sheet. - Mechanica, vol.47, pp.1131-1139.

[15] Madhu M. and Kishan N. (): Magnetohydrodynamic mixed convection stagnation-point flow of a power-law nonNewtonian nanofluid towards a stretching surface with radiation and heat source/sink. - Journal of Fluids Dx.doi.org/10.1155/2015/634186. 
[16] Hamad M.A.A., Uddin M.J. and Ismail A.I.M. (2012): Investigation of combined heat and mass transfer by Lie group analysis with variable diffusivity taking into account hydrodynamic slip and thermal convective boundary conditions. - International Journal of Heat and Mass Transfer, vol.55, No.4, pp.1355-1362.

[17] Olajuwon B.I. (2013): Effect of thermo diffusion and chemical reaction on heat and mass transfer in a power-law fluid over a flat plate with heat generation. - International Journal of Non-Linear Science, vol.15, No.2, pp.117127.

[18] Kishan N. and Shashidar Reddy B. (2013): MHD effects on non-Newtonian power-law fluid past a moving porous flat plate with heat flux and viscous dissipation. - International Journal of Applied Mechanics and Engineering, vol.18, No.2, pp.425-445.

[19] Postenicu A. (2004): Influence of magnetic field on heat and mass transfer from a vertical surfaces in porous media considering Soret and Dufour effects. - International Journal Heat and Mass Transfer, vol.47, pp.14671472.

[20] Mohammad M.R., Ali M., Behnam R., Peyman R. and Gong-Nan Xie (): Heat and mass transfer for MHD viscoelastic fluid flow over a vertical stretching sheet with considering Soret and Dufour effects. - Mathematical Problems in Engineering Dx.doi.org/10.1155/2015/861065.

[21] Pal D. and Chatterjee S. (2013): Soret and Dufour effects on MHD convective heat and mass transfer of a powerlaw fluid over an inclined plate with variable thermal conductivity in a porous medium. - Applied Mathematics and Computation, vol.219, pp.7556-7574.

[22] Wubshet Ibrahim and Bandari Shankar (2013): MHD boundary layer flow and heat transfer of a nanofluid past a permeable stretching sheet with velocity, thermal solutal slip boundary conditions. - Computers and Fluids, vol.75, pp.1-10.

[23] Hirschhorn J., Madsen M., Mastroberardino A. and Siddique J.I. (2016): Magnetohydrodynamic boundary layer slip flow and heat transfer of power-law fluid over a flat plate. - Journal of Applied Fluid Mechanics, vol.9, No.1, pp.11-17.

[24] Shashidar Reddy B., Kishan N. and Rajasekhar M.N. (2012): MHD boundary layer flow of a non-Newtonian power-law fluid on a moving flat plate. - Advances in Applied Science Research, vol.3, No.3, pp.472-1481.

[25] Bellman R.E. and Kalaba R.E. (1965): Quasi-Linearization and Non-linear Boundary Value Problems. - New York: Elsevier. 